\title{
COMING EVENTS
}

Events wholly or partially sponsored by IUGS, one of its Affiliated Associations or joint ventures - the International Geological Correlation Programme (IGCP) or the Inter-Union Commission on the Lithosphere (ICL).

- Signifies first announcement of meeting in EPISODES.

\section{6}

\section{Voveniber 1986}

ENGINEERING IN COMPLEX ROCK FORMATIONS (International Symposium), Beijing, P.R. China. Languages: English and Chinese. (Secretary of the licirt Symposium, Institute of Geoptysics, deadenis Sinicil, P.O. t3ox 928 , Beijing, P.R. (Chint)

Novenuer $1-4,1986$

INDIGENOUS RAW MATERIALS AND THEIR INDUSTRIAL U'TILIZATION IN THE GULF REGION (Conference), huwail. (Institute for Seientific Researeh, Goverment Relations Office, P.O. Box 24885 safat, huwait)

\section{Noveniber $2-5,1980$}

UNDERWATER MINING INSTITUTE (17th Hecting), Biloxi, Mississippi, U.S.A. (Wr. A. Willer, Lnderwater Wining Institute, University of Wisconsin Sea cirant, 1801 University Avenue, iladison, W1 53705 , U.S.A.)

\section{ivovember $2-6,1986$}

SOCIETY OF EXPLORATION GEOPHYSICISTS (56th Annual heeting), llouston, Texas, U.S.A. (Society of Exploration Geophysicists, 1'O. Box 3098, Tulsa, OK 74101 , U.S.A.)

November $6-8,1986$

STRATEGIES FOR EXPLOITATION OF IVINERAL RESOURCES IN DEVELOPING COUNTRIES (International symposium), Dhanoad, India. (Prof. A.k. Ghose, Indian school of Wines, !hanbad 826 but, India)

November $y-14,1986$

COASTAL ENGINEERING (International Conference), Taipei, lawan. (B.l. Edge, Cubit Engineering Linited, 207 East Bay street, Suite 311 , Charleston, $\$(29401$, (i.S.t.)

Vovember $10-11,1986$

* eXPloration GEochemistry (Irternational South buropean Symposium), Athens, Greece. Co-sponsored by $\mathrm{ABG}$. (Organizing Committee, International south European Symposium in Exploration Geochemistry, Institute of Gology and Mineral Exploration, 70 Nessoghion Street, 11527 Athens, Greece)

November $10-13,1986$

GEOLOGICAL SOCIETY OF AMERICA (Annual Meeting), San Antonio, Texas, U.S.A. (heetings Department, GSA, P.O. Box yl40, Boulder, co 80301 , U.S.A.)

November $10-15,1986$

SUBDUCTION ZONES (International kaiko Conference), Tokyo and Shimizu, Japan. (K. Kobayashi, Ocean Research Institute, University of Tokyo, 1-15-1 Minamidai, Nakano-ku, Tokyo 164, Japan)

November $17-18,1986$

GEOTHERMAL ENERGY (Weeting), San Diego, California, U.S.A. (Geothermal Resources Council, Box 1350, Davis, California y5617, U.S.A.)

November $17-21,1986$

MINING LATIN AMERICA (International Conference), Santiago, Chile (The Conference office, institution of Wining and hetallurgy, 44 Portland Place, London WIN $4 \mathrm{BBR}$, U.K.)

November $23-27,1986$

ARGENTINE PALAEONTOLOGY AND BIOSTRATIGRAPHY CONGRESS (4th), Mendoza, Argentina. (Dr. W. Volkheimer, Pribipa-Cricyt-CC $131, \quad 5500$ Wendoza, Argentina)
- IGCP-210 (Working (iroup Heeting), los, Nigeria. (C... Kogbe, Rock View Internat tional, $1-5$ rue ciutenberg, 75015 Paris, liance)

Noveriber $26-28,1986$

REE, NB AND TA ORE DEPOSITS (Workshop), Berlin, F.R.G. (Yrof. P. Möller, ltahn-Neitner Institut für hernfoischung, P.O. Box 390128 , Gilenteker Strasse 100, D-1000 Berlin $3 y$, l. .R.G.)

November $27-28,1986$

^ MICROCOMPUTERS IN EXPLORATION AND DATA INTERPRETATION (COGEODATA Workshop), Paris, Erance. (Lr. Ci. Gabert, Federal Institute for (ieosciences and $\mathrm{Natural}$ Resources, P.O. Box 51 al 53 , 1)-300u 1 annover 51 , Federal Republic of Germany)

1)ecember $1-4,1986$

COMPUTER AIDED DESIGN AND MONITORING IN GEOTECHNICAL ENGINEERING (16th Geotechnical Engineering Symposium), Bangkok, 'Theiland. (A.S. Balasubramaniam, GTE Division, P.O. Box 2754, Bangkok 10501, Thailand)

Uecember $1-5,1986$

- NEW ZEALAND GEOLOGICAL SOCIETY (Annual Conference), Palmerston North, New Zealand. (Geology Department, Univ. Canterbury, l'rivate Bag, Christehureh, New Zealand)

December $1-5,1986$

RESEARCH IN GEOPHYSICS AND GEOPHYSICAL EXPLORATION IN AFRICA (International Conference), Kano, Nigeria. Co-sponsored by International Lithosphere Program. (ACikA Conterenee, c/o vept. of Physies, (iniversity of Jos, Jos, Nigeria)

NEOTECTONICS AND VOLCANIC HAZARDS (International Symposium), Bogota, Colombia. Co-sponsored by A(all, languages: Spanish and English. (Dr. J.E. Valdiri W., P.O. Box 4865, Bogota, Colombia)

December $2-3,1986$

EARTHQUAKE ENGINEERING 1986: EARTHQUAKE RISK IN THE AUSTRALASIAN AND SOUTHWEST PACIFIC REGION (Seminar), Sydney, Australia. (Conference Vianager, Earthquake Engineering 1986, the Institution of Engineer's, Australia, 11 National Circuit, Barton ACT 2600, Australia)

December $2-4,1986$

HYDRODYNAMICS AND SEDIMENT DYNAMICS OF TIDAL INLETS (Symposium), Woods Hole, ivass., U.S.A. (D.G. Aubrey, WHOI, woods Hole, 1402543 , L.S.A.)

December $2-4,1986$

- construction and display of GeoSCIENTIFIC MAPS DERIVED FROM DATABASE (Symposium), Dinkensbühl, F.R.G. Cosponsored by COGEODATA. (Dr. G. Gabert, Federal Insitute for Geosciences and Natural Resources, P.O. Box 51 0l 53, 5-3000 llannover 51, Federal Republic of Germany)

December 4 - 10, 1986 REMOTE SENSING OF ENVIRONMENT (12th International Symposium), Nairobi, Kenya. Languages: English and French. (Remote Sensing Center, linvironmental Research Institute of Michigan, P.O. Box 8618, Ann Arbor, NI 48107-8618, U.S.A.)

December $5-7,1986$

GEOLOGY OF INDOCHINA (Conference), Ho Chi winh City, Vietnam. (Conference Secretariat CGI, General Department of Geology, 6 Pham Ngu I.ao St., Hanoi, Vietnam)
November $24-30,1$ y86

\section{vecember $1-6,1986$}

Decomber $6-13,198 \%$

- water resources neEds and pLanNING IN DROUGIIT-PRONE AREAS (international Conference), hhartoum, Sudan. (1)r. I..k. . kitgid, Departunent of Civil Engineering, Khartoum University, 1.0. Box 321, Khartoum, Sudan

December $7-12,1986$

ARCHAEOLOGICAL GEOLOGY: ENVIRONMENTAL SITING AND MATERIAL USAGE (GSi Penrose conferenee), Saint simons Islatrd, Georgia, U.S.A. (C.J. Vitaliano, vepartment of Geology, indiand University, 1005 Jast renth Street, Bloonington, IN 47405 , (.S.A.)

Vecember $8-12,198+5$

- SURFACE MINING;, HYDROLOGY, SEUIMENTOLOGY, AND RECLAMATION (I986 Symposium), Lexington, Kentucky, U.S.A. (J. Snedegar, 223 Transporation Research Building, University of Kentucky, Lexington, hY $40506-0043$, U.S. A.)

December 8 - 12, 1986

AMERICAN GEOPHYSICAL UNION (Fall Heeting), San Franeiseo, California, U.S.A. AaU Heetings, :040 Florida Avenue $\mathrm{NW}$, Washing ton, be 20009 , U.S.A.)

December $10-12,1986$

JAPAN EARTHQUAKE ENGINEERING SYMPOSIUM (7th) Tokyo, Japan. languages: Japanese and English. (Omanizing Committee, 7 th Japan Earthquake Engineering Symposium, 5-2b-20 Shiba, Minato-ku, Tokyo, Japan)

vecember $10-12,19 \times 6$

EARTH SCIENCES IN THE SERVICE OF AFRICA'S DEVELOPMENT (ANSTI Kegional Seminar), Dakar, Senegal. (K.F. Seddoh, Université du Benin, BP 1515 , Lomé, rogo, or 1.O. Nyambok, Dept. of Geology, University of Nairobi, Box 30197, Nairobi, Kenya)

J)ecember $15-17,1986$

PHYLOGENY AND EVOLUTIONARY BIOLOGY OF ECHINODERMS (Weeting), London, U.K. (i)r. A.B. Smith, l)epartment of Palacontology, British Wuseum (Natural History), Cromwell Road, London SW7 5BL, U.h.)

December $15-20,1986$

* THE ROLE OF GEOLOGY IN URBAN DEVELOPMENT IN SOUTHEAST ASIA (Land-plan III - A Symposiumi), Hong Kong. Sponsored in part by IUGS, IAEG and AGIJ). (Geological Society of Hiong fiong, c/o Department of Geography and Gcology, University of Hong Kong, Pokfulam Road, Hong Kong)

December $29-31,1986$

EARTHQUAKE ENGINEERING $(8 \mathrm{th}$ Symposium), Roorkee, India. (Indian society of Earthquake Technology, Prof. B.V. Lavania, Department of Earthquake Engineering, Roorkee 247667 , india)

\section{7}

\section{January 1987}

GEOGRAPHIC INFORMATION SYSTEMS IN ASIA (IGU Regional Conference), Bangkok, Thailand. (D.F. Narble, Dept. of Geography, SLNY at Buffalo, Amherst, NY 14260 , U.S.A.)

January $5-9,198$ ?

METEORITES AND THE EARLY SOLAR SYSTEM (Conference), Tueson, Arizona, U.S.A. (Dr. J. Kerridge, Institute of Geoplyysics, University of (California, Los Angeles, CA 90024, U.S.A.)

January $6-8,1987$

- WATER FOR MANKIND (International Symposium), Cairo, Egypt. (NUURS - France, 127 boulevard Saint-Michel, 75005 Paris, France) 
january $7-23,1987$

GEOCHEMISTRY OF HYDROTHERMAL OREFORMING PROCESSES (NATO Advanced Study Institute), Nadrid and Salamanca, Spain. (H.L. Barnes, 235 Deike Building, Pennsylvania State Univ., University Park, PA 16802 , U.S.A.)

January $8-10,1987$

MAGMATISM IN THE OCEAN BASINS (Weeting), Leicester, U.K. (A.L). Saunders, Department of Geology, The University, Leicester LE1 7RH, U.K.)

!anuary $9-14,1987$

THE PALAEOENVIRONMENT OF EAST ASIA (2nd lnternational Conference), Hong kong. (Dr. E.K.Y. Cher/Dr. R.O. Whyte, Centre of Asian Studies, Univ, of llong Kong, tiong kong)

fanuary $19-25,1987$

HOW VOLCANOES WORK (llawaii Symposium), Hilo, Hawail. (Robert Decker, U.S. Geological Survey, MS-910, 345 Niddlefield Roud, Menlo Park, CA 94025, U.S.A.)

January $21-31,1987$

* GRANITES AND ASSOCIATED MINERALIZATIONS (International Symposium), Salvador, Bahia, Brazil. Languages: English, French and Portuguese. (ISGAN, Augusto J. Pedreira, SME- CPM: Kua Ceara, 3-Pituba, 41),000, Salvador, Bahia, Brazil)

january $25-31,1987$

- DESERT DEVELOPMENT (Weeting and Field Trips), Cairo, Lgypt. (A. Bishay, Desert Development Center, American University in Cairo, Box 2511, Cairo, Egypt)

January $27-30,1987$

CANADIAN REEF RESEARCH (Symposium), Banff, Aberta, Canada. (Canadian Reef Research Symposiun, University of Calgary Conference Office, 2500 University Drive $\mathrm{NW}$, Calgary, Alberta, Canada T2N (N4)

\section{January $27-30,1987$}

NATURAL DISASTERS (International Conterence), Havana, Cuba. (Palacio de las Convenciones, Apartado 16046 , Zona 16 , La Habana, Cuba)

Pebruary $2-4,1987$

- PACIFIC RIM COAL (2nd International Conference), llong Kong. (11. Baisden, Pasha Publications, 1401 Wilson Boulevard, Suite 910 , Arlington, VA 22207 , U.S.A.)

\section{Pebruary $2-6,1987$}

ASH: A NEW RESOURCE, (Symposium), Prctoria. (Dr. R.A. Kruger, CSIR-Frd, POB 395 , Pretoria 0001 , South Africa)

pebruary $2-6,1987$

- DEFORMATION OF CRUSTAL ROCKS (International Conference), int. Buffalo, NE Victoria, Australia. (Dr. D. Gray, Department of Earth Sciences, Nonash University, Clayton, Victoria 3168 , Australia)

February $7-12,1987$

QUATERNARY SEDIMENT'S IN THE ARABIAN GULF AND THE MESOPOTAMIAN REGION (International Conference), kuwait. (Conference Secretary, Quaternary Sediments 1987, Dept. of Geology, Kuwait University, Box 5969, Code 13060, Kuwait)

February $9-11,1987$

SINKHOLES AND THE ENVIRONMENTAL IMPACTS OF KARST (2nd Multidiseiplinary Conference and Field Trip), Orlando, Florida, U.S.A. (Dr. B.F. Beck, Florida Sinkhole Research institute, University of Central Florida, Orlando, FL 32816 , U.S.A.)

February $9-13,1987$

^ COMMISSION FOR THE GEOLOGICAL MAP OF THE WORLD (CGivW Plenary Assembly), Paris, France. (Secretary General, CGNi, 77 rue Claude-Bernard, 75005 Paris, France)

February $13-21,1987$

LOESS (3ro International symposium), New Zealand. (Dr, D.N. Eden, N.Z. Soil Bureau, DSIR, Private Bag, Lower Hut, New Zealand) liebruary $16-20,1987$

- in'teraction between sedimen'ts and WATER (4th International Symposium), Welbourne, Australia. (Dr. B.T. Hart, Water Sciences Center, Chisholm Institute of Teehnology, P.O. Box 197, Caulfield East, Victoria 3145, Australia)

February $23-26,1987$

- URBAN HYDROGEOLOGY AND CONTAMINATION OF AQUIFERS (Latin American Conference), Cochabamba, Bolivia. Languages: Spanish and Portuguese, but papers in French and English accepted. (Secretaria de la Conferencia, c/o V. Ricaldi, Casilla 183 , Cochabamba, Bolivia)

Eebruary $24-26,1987$ GEOSYNTHETICS 187 (Conference), New Orleans, Louisiana, U.S.A. (IFAI, 345 Cedar Building, Suite 450, St. Paul, wiN 55101 , U.S.A.)

\section{February $26-27,1987$}

- EROSION CONTROL ASSOCIATION (18th Antual Conference), Sparks, Nevada, U.S.A. (International Erosion Control Association, P.O. Box 195, Pinole, CA 94564-0195, U.S.A.)

Warch 1987

- Conjunctive use OF SURFACE AND GROUNDWATER FOR AGRICULTURE (International Symoosium), Lahore, Pakistan. (Dr. N.u. Awan, Centre of Excellence in water Resources Engineering, University Engineering and 'Technology, Lahore-31, Pakistan)

Wareh $3-4,1987$

- INVERSION TECTONICS (Mecting), London, U.K. (IVI. Cooper, British Petroleum, Britannic House, Noor Lane, London EC2Y 9BU, U.K.)

March $4-7,1987$

$\star$ DETACHMENT AND SHEAR $(77 \mathrm{th}$ Annual Meeting of the Geologische Vereinigung), Basel, Switzerland. Sponsored by International Lithosphere Program. (Prof. D. Bernoulli, Geologisches Institut, Univ. Basel, Bernoullistrasse 32 , (11-4056 Basel, Switzerland)

warch $7-15,1987$

‡•ESTIMATION OF NATURAL RECHARGE OF GROUNDWATER (international workshop), Antalya, Turkey. Co-sponsored by IAH. Language: English. (Dr. I.E. Seyhan, Free University of Amsterdam, Jepartment of
Earth Sciences, P.O. Box 7161, 1007 MC Amsterdam, The Netheriands)

Warch 10-14, 1987

ORIGIN AND EVOLUTION OF PLANETARY AND SATELLITE A'TMOSPHERES (Conference), Tueson, Arizona, U.S.A. (S.K. Atreya, University of Michigan, Space Research Building, Ann Arbor, NiI 481 09-2143, U.S.A.)

Niaren $11-12,1987$

- MINERAL RESOURCES RESEARCH IN THE USGS (3rd Annual NeKelvey Forum), Benver, Colorado, U.S.A. (Buhler and Abraham, Inc., 10102 Mekinney Aventue, Silver Springs, Neryland 20902 , U.S.A.)

March $16-20,1987$

- GEOCHEMISTRY OF WATERS IN DEEP SEDIMENTARY BASINS (GSA Penrose Conference), Oxnard, California, U.S.A. (L. Elms, Western Experience, 2369 Carriage Circle, Oceanside, CA 92056, U.S.A.)

Niarch $16-20,1987$

LUNARY AND PLANETARY SCIENCE (18th Conference) Houston, Texas, U.S.A. (Lunar and Planetary Institute, 3303 NASA Road 1 , Houston, TX 77052 , U.S.A.)

Warch $18-19,1987$

EARLY TERTIARY VOLCANISM AND THE OPENING OF THE NE ATLANTIC (Vleeting), London, U.K. (A.C. Morton, British Geological Society, Keyworth, Notts. NGl2 5 (GG, U.K.)

ivarch $23-24,1987$

EXTRACTIVE INDUSTRY GEOLOGY 187 (Meeting), Keele, Staffordshire, U.K. (Confer- ence office, liwh, 44 lortland Place, London WIN $4 \mathrm{BR}$, U.K.)

March $23-26,1987$

- SMALL MINE ECONOMICS AND DEVElOPMENT (Conference), London, U.K. (Small mine economics and development conference, International $\mathrm{klining}$, Cii House, 31 Theobalds Road, London WC 1 , U.K.)

March $23-28,1987$

GROUNDWATER MONITORING AND MANAGEMENT (International Symposium), Desden, G.D.R. Languages: English and Russian. (Ur. P. Lösel, Institut für Wasserwurtschaft, Schnellerstrasse 140, DDR-1190 Berlin, German Democratic Republic)

March $26-27,1987$

THE PHYLOGENY AND CLASSIFICATION OF THE TETRAPODS (Special Weeting), London, U.K. (il. Benton, Departiment of Geology, 'The Queen's University of Belfast, Belfast, BTr IN N, Northern Ireland)

Mareh 29 - April 3, 1987

- EROSION AND DEPOSITION WITH EMPHASIS ON SEMIARID AND ARID ENVIRONMENTS (Neeting), Jerusalem, Beersheba, Elat, Israel. Co-sponsored by INQUA. (Prof. A. Yair, Department of Physical Geography, Insitute of Earth Sciences, Hebrew University, 91904 Jerusalem, Israel)

Warch 30 - April 3, 1987

* VULNERABILITY OF SOIL AND GROUNDWATER TO POLLUTANTS (International Conference), Noordwijkatan Zee, 'The Netherlands. Co-sponsored by LAH. (VSPG $187 \mathrm{c} / \mathrm{o}$ Klvl, P.O. Box 30424, 2500 Gits The llague, The Netherlands)

Warch $30-$ April 4, 1987

- ICE-CORE ANALYSIS (Symposium), Bern, switzerland. (Mrs. 11. Richardson, International Glaciological society, Lensfield Road, Cambridge CB2 IER, U.K.)

April 1987

- GEOCHEMISTRY AND MONITORING IN REPRESENTATIVE BASINS (International Meeting), Prague, Czechoslovakia. (Dr. B. Molden, Geological Survey, Malostranskeran 19, 11821 Prague 1, Czechoslovakia)

April $1-3,1987$

• SEDIMENTOLOGY (8th IAH Regional veeting), Tunis, Tunisia. (Pr. Ali M'Rabet, f'aculté des Sciences de Tunis, vépartement des Sciences de la Terre, Campus Universitaire, 1060 Tunis, funisia)

April l - 10, 1987

- Geotechnical ENGINEERING AND haZARD ASSESSMENT IN NEOTECTONIC TERRAINS (Sino-British Conference), Tuiwan. (ur. J. Rowbotham, Department of cieological Sciences, University College, Giower Street, London WC1E 6BT, U.K.)

April $2-3,1987$

- MICROPALAEONTOLOGY, PALYNOLOGY AND PETROLEUM EXPLORATION, ON- AND OFFSHORE EUROPE (Weeting), Aberdeen, Scotland, U.K. (Geological Society, Burlington House, Piecadilly, London WIV UJU, U.K.)

April $6-10,1987$

HYDROLOGY IN PERSPECTIVE (International Symposium), Rome, Italy. Co-sponsored by Unesco, WhO, and lAlis. (International Association of Hydrological Sciences, GIBI s.a.s. Studio Congressi, Via Mareo Besso, 40, 00191 Rome, italy)

April 6-12, 1987

- LATE QUATERNARY SEA LEVEL: THE MARINE AND TERRESTRIAL RECORD (GSA Penrose Conference), Ferry Reach, Bermuda. (J.L. Carew, Department of Geology, College of Charleston, Charleston, SC 29424 , U.S.A.)

April $7-8,1987$

PHANEROZOIC IRONSTONES AND RELATED DEPOSITS (International symposium), 


\section{Coming Events}

Shetheld, L.k. (1)r. (i. Taylor, Dept. Science, Luton, Clle, park Square, luton LUI 3.JU, U.K.)

April $7-10,1987$

DRILLEX 87 (Internationa] Lxhibition and Conference on 1)rilling), Stoneleigh, Warwiek shire, U.k. TThe Conference Office, The Institution of ilining and vetallurgy, 44 Portland Place, London WIN 4BR, U.K.)

April $10-11,198 \%$

* RECONSTRUCTION AND CORRELATION OF THE PHANEROZOIC LACUSTRINE RECORD (IGCP-2l9 Workshop), Lake Lazern, Switzerland. (1)r. k. Kelts, EAw AG-Geology, Cll-8600 Iübendorf $2 \mathrm{H}$, Switerland)

April $13-15,1987$

* ARGENTINE PALAEOBOTANY AND PALYN OLOGY (7th Symposium), Buenos Aires, Argentina. Sinulaneous meetings by ICC.P 242 and [GCP-237. (Prof. W. A. Caccavari, M.A.C.N. "B. Rivaduvia", Av. angel ciallardo 470, Buenos Aires 1405, Argentina)

April $13-16,1987$

EUROPEAN UNION OF GEOSCIENCES (IV Biennial Conference), Strasbourg, France. (W. Lowrie, Inst. für cieophysik, HPP P5, E? Hönggerberg, CH 8093 Zurich, Switzerland)

April $13-16,1987$

*-ENVIRONMEN'TAL RECORDS FROM LACUSTRINE BASINS (ICCP-2] 9 Symposium at EGU) Strasbourg, France. (Ur. K. Kelts, tiW Geology, CH-8600 bübendorf Z.1, Switzerland).

April $21-22,1987$

- DELTAS: SITES AND TRAPS FOR FOSSIL FUELS (Geological Society veeting), London, U.K. (Geological Society, Burlington House, Piccadilly, London WIV UdU, U.K.

April 22-24, 1987

GEOLOGICAL KINEMATICS AND DYNAMICS, FROM MOLECULES TO MANTLE (International heeting), Uppsala, Sweder. (The GKi) Committee, Institute of Geology, Uppsala University, Box 555, S-75l 22 Uppsala, Sweden)

April $23-26,1987$

* international Geochemical eXPLORATION (12th AEG Symposium) and METHODS OF GEOCHEMICAL PROSPECTING (4th IAGC Symposium), Orléans La Source, France. (The Organizing Committee, 12th lGES - 4th Silile, BRGill, B.P. 6009 45060 Orleans Cedex, France)

April 26 - May 1, 1987

WORLD PETROLEUM CONGRESS (12th) llouston, Texas, U.S.A. (12th WHC Association, c/o American petroleum Institute, 1220 L Street NW, Washington WC 20005 , U.S.A.)

Aprii $27-29,1987$

LATE CENOZOIC PALEOENVIRONMENTS AND GEOLOGY OF THE ARCTIC (workshop), Spidsbergseter Pjelistue, Norway. (Dr. $\Lambda$. Elverh $\phi$ i, Norwegian Polar Research institute, P.O. Box 158, 1330 Oslo Lufthavn, Norway)

April 28 - Way 7,1987

ZECHSTEIN: STRATIGRAPHY-PALEOGEOGRAPHY-GEOCHEMISTRY (International Symposium), Hannover, F.R.G. (J. Lepper, Niedersächsisches Iaandesamt für Bodenforschung, P.O. Box 51 ol 53, L-3000 Hannover 5i, F.R.G.)

May 1987

EXPOSED CROSS SECTIONS OF 'THE CONTINENTAL CRUST (GSA Penrose Conference), southeastern Ontario, Canuda. (L.M. Fountain, Department of Geology, and Geophysics, University of Wyoming, Laramie, WY 82071 , U.S.A.)

May $3-8,198 ?$

THE CONSTRUCINON OF GEOLOGICAL CROSS SECTIONS: TECHNIQUES, ASSUMPTIONS, AND METHODS (GSA Penrose Conference), New Paltz, New York, U.S.A. (P.A.
Geiser, Jepartment of Geology and Geophysics, University of Connecticut, Storrs, U 06268, U.S.A.)

viay $3-7,1987$

ENGINEERING GEOLOGICAL ENVIRONMENT IN MOUNTAINOUS AREAS (International Symposium), Beijing, P.R. China. (Geological Society of China, hinistry of (ieology, Pai Wan chung, Fuchengmenwai, beijing, P.R. China)

Nay $3-8,1967$

GLACIAL FACIES MODELS (GSA perrose Conferenee), Southern Ontario, Canada. (I) $\mathrm{r}$ N. Eyles, Jepartment of Geology, Scarborough Campus, University of Toronto, Scarborough, ontario, candadivile (A4)

Way $10-15,1987$

- IMPACT OF VLBI ON ASTROPHYSICS AND GEOPHYSICS (Symposium), Cambridge, hass. U.S.A. (J. Moran Center for Astro-physies, Vail Stop 42, 60 Garden Street, Cambridge, WA 023,38 , U.S.A.)

May $12-14,1987$

COASTAL SEDIMENTS 187 (Conference), New Orleans, Louisiana, U.S.A. (Dr. N.C. hraus, USAE Waterways lixperiment Station, Box 631, Attn: wLSCL-P, Vicksburg, is 39180-0631, U.S.A.)

May $15-17,1987$

- IIYDROLOGY, SEDIMENTOLOGY, AND GEOMORPHOLOGICAL IMPLICATIONS OF FLOODS (Conference), Lancaster, U.K. (P.A. Carling, rreshwater Biological Association, The Ferry House, far Sawrey, Ambleside, Cumbria LA22 (ILP, U.K.)

May $16-24,1987$

$\star$ PALAEOECOLOGICAL - PALAEOHYDROLOGICAL STUDIES BASED ON STRATIGRAPHICAL RESEARCH IN LAKES AND MIRES, AND FLUVIAL ENVIRONMENTS (iGCP-158 Symposium), Sweden. (13.L. Berglund, Department of Quaternary Geology, T'ornav. 13, \$-223 63 Lund, Sweden)

Wuy $18-22,1987$

*GEOMATHEMATICS AND GEOSTATISTICS APPLIED TO SPACE- AND TIME-DEPENDENT DATA (International Confernce and Course), Wroclaw, Poland. Sponsored by CODATA, IAMG, IUGS, and Unesco. (Dr. J.J. Royer, C.R.P.G., B.P. 20, 15 rue Nd des Pauvres, 54501 Vandoeuvres-les-Nancy Cedex, France)

Vay $18-22,1987$

AMERICAN GEOPHYSICAL UNION (Spring Weeting), Baltimore, Maryland, U.S.A. (AGU Veetings, 2000 Florida Avenue NW, Washington, DC 20009, U.S.A.

vay $25-27,1987$

COASTAL LOWLANDS: GEOLOGY AND GEOTECHNOLOGY (International Symposium), The Hague, The Netherlands. (Dr. H.J.W.G. Schalke, P.O. Box 85947, 2508 CP The Hague, The Netherlands)

May $25-27,1987$

GEOLOGICAL, MINERALOGICAL ASSOCIATIONS OF CANADA (Joint Annual iveeting), Saskatoon, Canada. (Dr. W.O. Kupsch, bept. Geological Sciences, University of Saskatchewan, Saskatoon, Sask., Canada S7N OW()

Way $27-28,1987$

- ALPINE TECTONICS (Geological Society, The Fermor Lecture Vleeting), London, U.K. (Prof. N.P. Coward, Hepartment of Geology, hoval School of Nines, Imperial College, Prince Consort Road, London SW $2 \mathrm{BP}$, L.K.)

May $28-30,1987$

PERMANENT SEISMOGRAPHIC OBSERVATORIES AND NETWORKS (Centennial Anniversary symposium), Berkeley, California, U.S.A. (prof. B.A. Bolt, Seismographic Stations, University of California, Berkeley, CA 97420 , U.S.A.) lay 31 - June 5,198 ?

WORLD MINING CONGRESS (13th), Stockholm, sweden. (Organizing Seeretary, I3th world Mining Congress, University of Luled, S-45l 87 Lulea, Sweden)

June 1987

INTERNATIONAL MINING AND EXPLORATION EXHIBITION 187 (heeting), Sydney, Australia. (Thomson Exhibitions, 47 Chippet Street, Chippendale, NSw 2008, Australia)

June $3-6,1987$

* PROTEROZOIC GEOCHEMISTRY (Internit lional Symposiumi, Lund, Sweden. Sponsored by lGip-2l7. (Prof. R. Corbatsehev, Geo logical Insitute, land university Solvegatan $13,5-22362$ lund, sweden)

June $7-10,1967$

$A A P G$ and SEPM (Anual heeting), Los Angeles, Calif., U.S.A. (AAPG Headquarters, Box 979 , Tulsa, OH 74101 , U.S.A.)

June $y-12,1987$

- EUROPEAN ASSUCIATION OF EXPLORATION GEOPHYSICIS'IS (4yth Annual weeting and 'lectunical Exhibition), Belgrade, Yugoslavia. (F.AEu, Wassenaarseweg 22, 2596 CH The Hague, The Netherlands).

June $11-12,1987$

FAN-DELTAS: SEDIMENTOLOGY AND TECTONIC SETIINGGS (international Symposium), Berren, Norway. Sponsored by Norsk Hydro, Univ. of hergen and Norwegian Petrolcun Sociely. (R.J. Steel, Nor'sk Hydro Researeh Centre, P.O. (30x 4.314, 5013 Bergen, Norway)

June $21-25,1987$

- HYDROGEOLOGY (4th Canadian/American Conferenee), Banff, Aberta, Canada. (Dr. 13. Hitchon, Alberta Researeh Council, P.O. Box 8330, Station F, Ldmonton, Alberta, Canada (6) $15 \times 2)$

June $30-$ July $b, 198 ?$

$\star \bullet C H E M I S T R Y$ OF THE EARTH AND 'THE UNIVERSE (IAC 20th Anniversary Congress), Paris, France. (1)r. B. Hitchon, Alberta Research Council, P.O. Box 8330 , Station F, lidmonton, Alberta, Candda Tb11 $5 \times 2$ )

duly $6-10,1987$

* CRYPTOEXPLOSIONS AND CATASTROPHES IN THE GEOLOGICAL RECORD (International Workshopl, Parys, South Africa. Co-sponsored by IUCS. (1.O. Nicolaysen, Geophysies bepartment, Witwatersrand Lniversity, Johannesburg, South A frica 2001)

July $6-11,1987$

CONTINENTAL AND OCEANIC LITHOSPHERE: SIMILARITIES AND DIFFERENCES (Workshop), London, U.K. (ki.A. Nenzies, bepartment of Geology, University of London, RIIBNC, Egham, Surrey IW20 0EX, U.K.)

July $6-11,1987$

- FOSSIL ALGAE (4th international Symposiun), Cardiff, Nales, L.K. (I)r. K. Kiding, bepartment of Gology, University College, Cardiff CFl LXL, Wales, L.K.)

July $7-10,1987$

APPLIED MINERALOGY (3rd International Congress), Orléans, France. (P'. Alain, ICA 87, Laboratoire de Winéralogie Appliquée, Ecole sup. de l'linergie et des ivatériaux, Domaine Universitaire de la Source, B.t. 6749 , 45067 Orléans, Cedex 2, irance)

July $23-25,1987$

- SOUTH ATLANTIC EVOLUTION (2nd symposium), Rio de Janeiro, Brazil. (D. Dias-Brito, PETRULKRAS/CENPES, llha do Fundão, Quadra 7 , Rio de Janeiro 21.910 , Brazil)

July $27-30,1987$

LATIN AMERICAN PALEONTOLOGY $(4 \mathrm{th}$ Congress) Santaz Cruz de la Sierra, Bolivia ( $D r$. h. Seiarez-Rigl, Congress de Paleontoloria, Casilla \#1321, Santat (ruz de la Sierra, Boliviat) 


\section{Coming Events}

iuly $29-31,1987$

* PACIFIC NEOGENE STRATIGRAPHY (4th International Conoress of Regional Committee and Yeeting of IGCP 246), Berkeley, Calif, U.S.A. (Dr. C. Brunner Department of Paleontology, University of California, Berkeley, CA 94720, U.S.A.)

July 31 - August 9,1987

* international union for quater NARY RESEARCH (12th Congress), Ottawa, Ontario, Canada. (Dr. Alan V. Morgan, Department of Earth Sciences, University of Waterloo, Waterloo, Ontario, Canada N2L $3 \mathrm{Gl}$ ) ugust $1-12,1987$

- LANDSLIDES (5th International Conference Workshop), Australia and New Zealand. (5th ILFL-Anzslide '87, P.O. Box 56, Rosny Park, Tasmania 7018 , Australia)

\section{lugust $3-7,1987$}

EROSION AND SEDIMENT TRANSPORT IN PACIFIC RIM MOUNTAINOUS LANDS (International Symposium and IALIS/LUGG Meeting), Corvallis, Oregon, U.S.A. (Conference Coordinator, College of Forestry, Oregon State University, Corvallis, OR Y7331, U.S.A.)

?ugust $3-13,1987$

- MAGMATIC SULPHIDES (5th International Sulphides Conference), Harare, Zimbabwe. (Secretary, 5th International Sulphides Conference, Box 1795, Harare, Zimbabwe)

ugust $9-11,1987$

•-TRACE FOSSILS, SMALL SHELly fOSSILS, AND THE PRECAMBRIAN-CAMBRIAN BOUNDARY, ST. JOHN'S, NEWFOUNDLAND (IUGS Commission on Stratigraphy Conference and Fieldtrip), eastern Newfoundland. (Dr. G.M. Narbonne, Department of Geolugical Sciences, Queen's University, Kingston, Ontario, Canada K $7 L, 3 \mathrm{~N} 6$ )

ugust $9-18,1987$

* PRECAMBRIAN-CAMBRIan BOUNDARY WORKING GROUP (Weeting), St. John's, Newfoundland, Canada (Dr. G. Narbonne, Department of Geological Sciences, Queen' University, Kingston, Ont., Canada, K7L 3N6

August $9-22,1987$

IUGG (XIX General Assembly), Vancouver, Canada. (R.D. Russell, IUGG, Department of Geophysies and Astronomy, University of British Columbia, Vancouver, B.C., Canada V6' (W5)

ugust $y-22,1987$

* the physical basis of ICE SHeEt MODELLING (IAHS Symposium at IUGO General Assembly), Vancouver, B.C., Canada (E.1) Waddington, Cleophysics Program AK-50, University of Washington, Seattle, WA 98195 , U.S.A.)

igust $12-13,1987$

- Geotechnical ENGINEERING on soft SOLLS (International Symposium), Mexico City. (N. Mencoza, Chairman, Orwanizing Cormmittee, Instituto de Ingeniera - UNAM, Apdo. Postal 70-472, 0451 0 .Hexico, D.F., Wexico)

sugust $12-20,1987$

IN'TERNATIONAL UNION OF CRYSTALLOGRAPHY (Congress), Perth, Western Australia. E.N. Waslen, Crystallography Centre, University of Western Australia, Nedlands, 6009, Australia)

Jugust $17-20,1987$

DEVONIAN SYSTEM (2nd International Symposiuri), Calgary, Alberta, Canada. (Devonian Symposium, Canadian Society of Petroleum Geologists, 505-206 7th Avenue SW, Calgary, Alberta, Canada T2P 0W7)

ugust $17-21,1987$

- BASEMENT TECTONICS (7th International Conference), Kingston, Ontario, Canada. (Bob Vason, e/o Events Mlanagement Inc., 4 Cataraqui Street, Suite 209, Kingston, Ontario, Canada K7K 1Z7)
August $18-22,1987$

AFRICAN GEOLOGY (14th Colloguium) Berlin (West). (G. ilatheis, 'Technical University of Berlin, SRP/69, Ackerstrasse 71-76, D-1000 Berlin 65, F.R.(i.)

A ugust $20-22,1987$

X-RAY POWDER DIFFRACTOMETRY (Nieeting with 14 th Congress of the International Union of Crystallography), Perth, Western Australia, (Dr. E.H. Nickel, Division of Winerals \& Geochemistry, CSIRO, Private 13 ag PO, Wembley, W.A., Australia 6014)

A ugust $20-30,1987$

PACIFIC SCIENCE ASSOCIATION (16th Congress), Seoul, South Korea. Section B: Solic Earth Sciences. Language: English. (Prof Bong Kyun Kim, Dept. of Cieological Sciences, Seoul National University, Seoul, South Kiorea)

August $21-22,1487$

Ł-DEVONIAN SUBCOMMISSION (Open Mlceting), Calrary, Alberta, Canada. (i) r. W. A. Oliver, Jr., U.S. Geological Survey, E-305 Natural History Building, Smithsonian Institution, Washington, DC 20560, U.S.A.)

August $24-28,1987$

ANTARCTIC EARTH SCIENCES (5th International Symposium), Cambridge, U.K. (Dr. M.R.A. Thomson, British Antaretic Survey, High Cross, Nadingley Road, Cambridge, U.K. CB3 OET)

August $26-29,1987$

PACIFIC RIM CONGRESS 87 (International Congress), Gold Coast, Southern queensland, Australia. (Aus. Inin Congress Secretariat, P.O. Box 731 Toowong, 4066 Queensland, Australia)

A ugust 26 - September 7, 1987

CRETACEOUS SYMPOSIUin (3rod Inter national symposium), Tubingen, F.K.G. (Prof ur. J. Wiedmann, Institut und ivluseum für Geologie und Paläontologie, Sigwartstrasse 10 7400 'Tubingen 1, F ederal Republic of (jermany)

sugust 30 - September 4, 1987

INTERNATIONAL SOCIETY FOR ROCK MECHANICS (6th International Congress), treal, Canada. (Prof. B. Ladanyi, Dept. Civi Ëngineering, Ecole Polytechnique, Box 6079, Stn. A, Montréal, Canada $\mathrm{H} 3 \mathrm{C} 3 \Lambda 7$

A ugust 31 - September 2, 1987

- AFRICAN MINING (International Conference), Harare, Zimbabwe. (The Institution of Mining and Metallursy, 44 Portland Place, London WIN $4 \mathrm{BR}$, U.K.)

August 31 - September 3, 1987

SOIL MECHANICS AND FOUNDATION ENGINEERING (9th European Conference), Dublin, Ireland. Lenguages: English and French. (Dr. Trevor Orr, Civil Engineering Department, Trinity College, Dublin 2, Ireland)

August 31 - September 4, 1987

CARBONIFEROUS STRATIGRAPHY AND GEOLOGY (11th International Congress), Beijing, P. R. China. Languages: Chinese and English. (Prof. Yang Jing-zli, Nanjing Institute of Geology and Palaeontology, 39 East Beijing Road, Chi-ihing-Ssu, Nanjing, P.R. China)

August 31 - September 5, 1987

YELLOWKNIFE 187 (GAC Field Weeting), Yellowknife, NWT, Canada. (W.A. Padgham, Geological Surveys, Bag 9100, Yellowknife, NWT, Canada X1A 2R3)

September $1-5,1987$ AFRICAN GEOLOGY (14th Colloquium) Berlin, (West), F.R.G. (Dr. G. Watheis, Technical University of Berlin, SFB 69, ckerstrasse 71, D-1000 Berlin 65, F.R.G.)

September $6-12,1987$

* BASIN ANALYSIS (COGEODATA Workshop), Budapest, Hungary. (Dr. G. Gabert, Federal Institute for Geoseiences and Natural Resources, P.O. Box 5l 01 53, D-3000 Hannover 51 , Federal Republic of Germany)
September $7-9,1987$

DEFORMATION AND PLATE TECTONICS

International Conference), Oviedo, Spain. Language: English. (A. Perez-Estaun, Lpto de Geologia, Universidad de oviedo, 33005 Oviedo, Spuin)

September $7-12,1987$

ॠCOMPUTERIZED BASIN ANALYSIS (CO(iEOI)ATA International Workshop), Szeged, Hungary. Language: English. (J., L. Somos, Geologieal Survey of Hungary, Ph. 106, $\mathrm{H}-\mathrm{l}$ 442, Budapest, Hungary)

September $7-12,1987$

ANTARCTIC GLACIOLOGY (4th Internationa SCAR Symposium), Bremerhaven, F.R.G. Heinz Kohnen, Alfred Wegener Institute for polar Research, Columbus Center, L-2850 Bremerhaven, F.R.G.)

Septernber 8-14,1987

TERMINAL PRECAMBRIAN AND CAMBRIAN GEOLOGY (International Symposium) Yichang, China. Languages: Chinese and English. (Dr. Wang Xiao-feng, Terminal Precambrian and Cambrian Geology, Yichang Institute of Geology and ilineral Resources, P.O. Box 502, Yichang City, Hubei Province, People's Republic of (China)

September $11-14,1987$

SEPM (4th Annual Widyear Vleeting), Austin Texas. (Stiph, P.O. Box 4756, Tulsa, OK 74154, U.S.A.)

September $11-17,1987$

- PALEOENVIRONMENTAL INTERPRETATION OF PALEOSOLS (GSA Penrose Conference), Warm Spring Indian Reservation, Oregon, U.S.A. (G.d. Retallack, Department of Geology, University of Oregon, Eugene, OR 97403, U.S.A.)

September $12-23,1987$

* COMPUTER APPLICATIONS AND MANAGEMENT OF PETROLOGICAL DATA BASES (Workshop), Kuwait. Co-sponsors inelude IUGS and IGCP-239. (Dr. Ali T. Al-ikishwt, Geology Department, Kuwait University, P.O. Box 5969 , Safat, Kuwait)

September $14-16,1987$

- THE ORIGIN OF GRANITES (Symposium), kdinburgh, Scotland, U.K. (The ileetings Secretary, The Royal society of Edinburgh, 22-24 George Street, Edinburgh Ef12 $2 \mathrm{PQ}$, Seothand, U.K.)

September $14-18,1987$

* ANDEAN VOLCANISM SYMPOSIUM (10th Arrentine (Geological Congress), San Salvador de Jujuy, Argentina. Co-sponsors inelude IAVCEI and IGCP-249. (1)r. B. Coira, CONICET-Univ. Nae de Jujuy, Casilla de Correo No. 258, 4600 San Salvador de Jujuy, Argentina)

September $14-18,1987$

NEOTECTONICS AND SEISMICITY OF THE ANDES (Regional Symposium), San illiguel de T'ucumán, Argentina. (Dr. V. Ramos, Department of Geology, University of Buenos Aires, Pabellon 2 - City University 1428, Nunez, Buenos Aires, Argentina)

September $14-18,1987$

CIRCUM-PACIFIC PHANEROZOIC GRANITES (International Symposium), Tucumán Argentina. lGCP-249 jointly with 101h Argentine Geological Congress. (Dr. Carlos W. Rapela, Centro de Investigaciones Cieologicas, Universidad Nacional de La Plata, Calle 1 no 644, 1900 La Plata, Argentina)

September $14-18,1987$

$\star$ HYDROGEOLOGY OF COAL BASINS (IUGS/IAH Symposium), Katowice, Poland. Dr. A. Rozkowski, Geological Institute, Bialego 1, 41-200 Sosnowiec, Poland)

September $17-27,1987$

* EVOLUTION OF METAMORPHIC BELTS (Geological Society and IGCP-235 Joint Meet 


\section{Coming Events}

ing), Dublin, Irctand. (J.s. Daly, Department of (ieology, University College, Belfield Campus, bublin 4, Ireland)

Scptember $21-25,1987$

- NatTuRal Gilasses (imeeting), Prague, Czechoshovakia. Language: I'nglish. (V. Bouska, liaculty of Science, Charles University, Albertov 6, 12843 Prague 2 , (zechoslovakia)

September $21-25,1987$

- METALS AND METALLOIDS IN THE HYDROSPHERE: IMPACT THROUGH MINING AND INDUSTRY, AND PREVEN'ITON TECHNOLOGY (Unesco/IH I International Workshop), Bochum, F.R.t. (IH $1 \mathrm{p} / \mathrm{OHP}$ Sekretariat, c/o Bundesanstalt für Gewässerkunde, Postfach 309, D-5400 hoblenz, R.R.(G.)

September 24 - Oetober 1, 1987

* FOSSILS, ROCKS AND HISTORY (13th INHUGEO Symposium), Pisa, Italy. (Prof. (A. Giglia, Dip. Seienze della llerra, Via s. Maria 53,56100 Pisa, I taly)

september $25-27,1987$

CATASIROPHIC FLOODING (Ibit Ammal Geomorphology Symposium), Oxford, Ohio, U.S.A. (Dr. Larry itayer, Department of Geology, viani Unversity, Oxford, Onio 45056, U.S.A.)

September 27 - October 1,1987

EXPLORATION 187 (3rd becennial) Conference on ucophysical and Geochemical Explo ration for Vinera!s and Ground-water (3ro becennial Conference), Toronto, Canada. (Exploration '87, c/o 222 Snidercroft Road, Conford, Ontario l.4K I [35, Canada)

Oetober $6-9,1987$

OROGENY, MAGMATISM AND METALLOGENY IN EUROPE (European Geological Societies 5th Meeting), Dubrovnik, Yugoslavia. Janguages: English and French. (European Centre for Peace and Development, Secretariat Mlios 5, Sava-Centre, P.O. Box 5, 11000 Beograd, Yugoslavia)

Oetober $11-15,1987$

SOCIETY OF EXPLORATION GEOPHYSICISTS (57th Annual Weeting), New Orleans, La, U.S.A. (Harvin R. Hewitt, Amoco Production Co., Box 591, Tulsa, OK 74102 , U.S.A.)

October $12-16,1987$

^ MATHEMATICAL METHODS IN GEOLOGY (16th Anmual Geochautaugua), Pŕíbram, Crechoslovakia. Co-sponsored by IAMG. (A. Ryčl, The vining Príbram, Box 4l, 26102 prúbram, Czechoslovakia)

October $1 y-23,1987$

- APPLICATION OF COMPUTERS AND MATHEMATICS IN THE MINERAL INDUSTRIES (20th International Symposium), Johannesburg, South Africa. (The Conference Secretary (C.31), Nintek, Private bag $\times 3015$ Randburg, 2125 South if frica)

October $26-2 y, 1987$

GEOLOGICAL SOCIETY OF AMERICA (Annual Weeting), Phoenix, Arizona, U.S.A. (Weetings Department, GSA, Box 9140 , Boulder, CO 80301 , U.S.A.)

Oetober 31 - November 3,1987

- PERovSKites: A STRUCTURE OF GREAT INTEREST TO GEOPHYSICS AND MATERIALS SCIENCE (AGU Chapman Conference), Bisbee, Ariz., U.S.A. (AGU Weetings, 2000 Florida Avenue NW, Washington, OC $200(19$, U.S.A.)

November 1987

•GEOLOGY OF SOMALIA AND SURROUNDING REGIONS (International weeting), Mogadishu, Somalia. Sponsored by lUcis. Languages: Somali, Arabic, English, Italian. Texts in English. (Dean of Geology Faculty, Somali National University, P.O.B. 1081, Mogradishy, Somala)
November 1987

* SOUTH american COgEOdata (Symposium), Ouro Preto, Brazil. (I)r. G. Gaberi, Federal Insitute for Geoseiences and Natural Resources, P.O. Box 51 ol 53 , D-3000 Hannover 51 , Federal Republic of (iermany)

Decenber $7-10,1987$

$\star$ TECTONOTHERMAL EVOLUTION OF WEST AFRICAN OROGENS (IGC P-233 international (Conference), Nouakchott, Maturitania. (R.L. Dallmeyer, Department of Geology, University of Georgia, A thens, Georgia 30602 , U.S.A.)

December $7-11,1987$

SOUTHEAST ASIAN GEOTECHNICAL CONFERENCE (9th) Bangkok, Thailand. Language: English. ('lhe Hon. Secretary, yth SEAGC, c/o Division of Geotechnical \& Iransportation Engineering, Asian Institute of l'echnology, P.O. Box 2754, Bangkok 14501 , Thailand)

Lecember $7-11,1987$

AMERICAN GEOPHYSICAL UNION (Fall leeting), San Francisco, California, U.S.A. $(A G \mathrm{U}$ Heetings, 2000 Florida avenue in. Washington, DC 20009 , U.S.A.)

\section{8}

January 31 - February 5,1988

- ACHIEVEMENTS IN AUSTRALIAN GEOSClENCE (9th Australian Geological Convention), Brisbane, Australia. (i)r. G.W. Hoffmann, Geologieal Survey of Queensland, GPO BOX 194 , Brisbane, queensland 4041, Australia)

V1 $\operatorname{arch} 8-11,1988$

ASIAN MINING 88 (3rd Internationa Conference and Exhibition), Kuala Lumpur, Malaysia. (The Conference Office, The Institution of vining and iletalurgy, 44 Portland lace, London WlN 4BR, U.K.)

Warch $20-23,1988$

AAPG/SEPM (Annual Vleeting), Houston, 'Texas, U.S.A. (Convention Department AAP' Box 974 , l'ulsa, OK 74101 , U.S.A.)

Vay $16-20,1988$

$\star$ BICENTENNIAL GOLD 88 (Conference), Melbourne, Australia. Cosponsored by Society of Economic Geologists. (Dr. R.R. Keays, Department of Geology, University of Melbourne, Parkville Vic 3052 , Australia)

Vay $16-20,1988$

AMERICAN GEOPHYSICAL UNION (Spring Neeting), Baltimore, Nlaryland, U.S.A. (AGU ineetings, 2000 Florida Avenue $\mathrm{NW}$, Washington, DC 20009 , U.S.A.)

Nay $16-20,1988$

* HYDROLOGICAL PROCESSES AND WATER MANAGEMENT IN URBAN AREAS (IAHS/ IU( $)$ G-IAH/IUGS-Uneseo Heeting), Duisburg, F.R.G. (Dr. E. Romijn, Provineial Waterboard of Gelderland, Narkstraat 1, P.O. Box 9090 , 6800 (iX Arnhem, The Netherlands)

May $22-25,1988$

- GAC/MAC/CSPG (Annual lileeting), St. John's, Newfoundland, Canada. (J.H. Fleming, Department of vines and Energy, P.O. Box 4750 , St. John's, N ew foundland, Canada A $1 \mathrm{C} 5 \mathrm{~T} 7$ )

May 29 - June 3,1988

WATER FOR WORLD DEVELOPMENT (6th IWRA World Congress), Ottawa, Ontario, Canada. (P.J. Reynolds, President, Canadian Committee - International Water Resources Association, 3 Valley View Road, Ottawa, Ontario, Canada K2H $5 \mathrm{Y}^{\prime} 6$ )

June $7-10,1988$

EUROPEAN ASSOCIATION OF EXPLORATION GEOPHYSICISTS (50th Congress), Den Haag, The Netherlands. ( $E$, van der Gaag, European Association of Exploration Geo physieists, P.O. Box 162, NL-2501 AN The Hague, The Netherlands)
July 1988

* APPLIED GEOCHEMISTRY OF THE CONTINENTAL CRUST (IAGC Conference), Sao paulo, Braxil. (1)r. A.d. Welfi, Institute of istronomy and Cieophysies, University of Sao l'aulo, C.P. 30627, Sao Paulo 01000, Brazil)

July 1988

- THERMODYNAMICS OF NATURAL PROCESSES (International Symposium), strasbourg, France. Co-sponsored by IAC:, IGCP, INA, CODAlla. (1)r. B. Fritz, Centre de Sédinientolorie et de ciéochimie de la Surface, 1 rue Blessig, $\mathrm{F}-62084$ Strasbourg Cedex, France)

July - August 1988

ఊ OSTRACODA AND GLOBAL EVENTS (10t1 International Symposium), Aberystwyth, Wales, L.K. Supported by IPA. (Dr. R.C. Whatley, Jeptrtuent of (Geology, University College of wales, Aberystwyth, Dyfed SY23 31)B, Walcs, U.K.)

July $1-8,1986$

SEISMIC PROBING OF I'HE CONTINENTS AND THEIR MARGINS (Symposium), canberra, sustralia. (Dr. J. Leven, Bill R, P.G. Box 378 , Canberra, Ac' 261t, Sustralia)

July $25-29,1988$

- FOSSIL CNIDARIA (5th litternational symposium), Brisbane, Australia. (I)r. J.S. Jell, bepartinent of Geology and Mineralogy, University of Queensland, St. Lueia, Queensland 4067 , Australia)

August $2-5,1988$

PERMAFROST (5th international Conference) Trondheim, Norway. (5th International Conference on Permafrost, Norwegian Road Research Laboratory, P.O. Box 6390 Etterstad, N-10604 Oslo 6, Norway)

August $14-21,1988$

- PEAT (8th International Congress), Leningrad, U.S.S.R. (Peat Congress, Winistry of Fuel industry of the RSFSR, Sadovaya-Chernogryazskaya 8, Moscow 107813 , U.S.S.R.)

August $20-27,1988$

INTERNATIONAL PALAEOBOTANICAL CONGRESS (3rd) Melbourne, Australia. Secretary, 3nd IOP Conference, Conventions Department, P.O. Box Iy0lR, G.P.O. inelbourne 3001 , Australia)

August $22-26,1988$

GEOGRAPHICAL CONGRESS (IGU $26 \mathrm{th}$ International), Sydney, Australia. (Prof. B. Thom, Jepartment of Geography, Institute Building, University of Sydney, Sydney, Australia 2006)

Aurust 28 - Septenber 2, 1988

INTERNATIONAL PALYNOLOGICAL CONGRESS (7th), Brisbane, Australia. (Dr. John Rigby, Conventions Department, P.O. Box 489, G.P.O., Syaney NSW 2001, Australia)

September 1988

* engineering Geology as RELATed to THE STUDY, PRESERVATION OF ANCIENT WORKS, MONUMENTS AND HISTORICAL SITES (IAEG International Symposium), A thens, Greece. (Оr, L. Primel, lAEG, Lab. Contral des Ponts et Chaussées, 58 Boulevard Lefebvre, 75732 Paris (edex 15, France)

September 1988

SNOW AND GLACIER RESEARCH RELATINC TO HUMAN LIVING CONDITIONS (Symposium), Norway. (Mrs. R. Richardson, Interna tional Glaciological Society, Lensfield Road, Cambridge CB2 lER, U.K.)

Wish to announce meetings of internationa significance? Send brochure or details to Episodes, Room 177, 601 Booth Street, Ottawa Canada $\mathrm{KlA} 0 \mathrm{E} 8$, at least one month in advanco of publication date. 